\title{
Efeitos gênicos de caracteres associados à eficiência no uso de nitrogênio em milho
}

\author{
Júlio César DoVale ${ }^{(1)}$, Roberto Fritsche-Neto(1), Felipe Bermudez ${ }^{(2)}$ e Glauco Vieira Miranda(1)
}

\begin{abstract}
(1)Universidade Federal de Viçosa, Departamento de Fitotecnia, Avenida P.H. Rolfs, s/no, CEP 36570-000 Viçosa, MG. E-mail: julio.vale@ufv.br, roberto.neto@ufv.br, glaucovmiranda@ufv.br (2)Universidade de São Paulo, Escola Superior de Agricultura Luiz de Queiroz, Departamento de Genética, Avenida Pádua Dias, oo 11, CEP 13400-970 Piracicaba, SP. E-mail: feliperamil@gmail.com
\end{abstract}

Resumo - Os objetivos deste trabalho foram determinar o controle genético da eficiência no uso do nitrogênio (EUN), identificar a importância das eficiências na absorção (EAN) e na utilização (EUtN) na sua composição, e quantificar relação entre produção de matéria seca da parte aérea (MPS) e do sistema radicular com a EUN e com seus componentes. Foram avaliadas 41 combinações híbridas em duas disponibilidades de N: baixa (BN) e alta (AN). Utilizou-se o delineamento de blocos ao acaso com duas repetições, em arranjo fatorial simples (combinação híbrida $\mathrm{x}$ disponibilidade de $\mathrm{N}$ ). As análises estatísticas foram realizadas por meio das equações de modelos mistos. Correlações de elevada magnitude foram detectadas entre EAN e EUN, bem como entre essas eficiências e a MPS, tanto em BN como em AN. Em ambas as disponibilidades de N, efeitos genéticos aditivos apresentaram maior importância para os caracteres associados à EUN. Dessa forma, a seleção baseada no desempenho individual de linhagens quanto à MPS pode possibilitar a obtenção de genótipos com alta EUN. Independentemente da disponibilidade de N, a EAN é o componente mais importante da EUN.

Termos para indexação: Zea mays, capacidade de combinação, disponibilidade de nitrogênio, estresse nutricional, REML/Blup, sistema radicular.

\section{Genetic effects of traits associated to nitrogen use efficiency in maize}

\begin{abstract}
The objectives of this work were to determine the genetic control of nitrogen use efficiency (NUE), to identify the importance of $\mathrm{N}$ acquisition (NAE) and utilization (NUtE) efficiencies on its composition, and to quantify the relation between production of shoot (SDM) and root dry matter mass with NUE and its components. Forty-one hybrid combinations were evaluated in two $\mathrm{N}$ availabilities: low (LN) and high $(\mathrm{HN})$. A randomized complete block design with two replicates, in a simple factorial arrangement (hybrid combination x N availability), was used. Statistical analyses were done using mixed model equations. High magnitude correlations were detected between NAE and NUE, and between these efficiencies and SDM in LN and HN. In both $\mathrm{N}$ availabilities, additive genetic effects were more important for the traits associated with NUE. Therefore, selection based on the individual performance of inbred lines as to SDM can allow for the obtainment of genotypes with high NUE. Independently of N availability, NAE is the most important component of NUE.
\end{abstract}

Index terms: Zea mays, combining ability, nitrogen availability, nutritional stress, REML/Blup, root system.

\section{Introdução}

Um dos desafios para os próximos anos é melhorar o aproveitamento do nitrogênio por meio do desenvolvimento de cultivares com alta eficiência no uso do mesmo (EUN). De acordo com Moll et al. (1982), a EUN consiste na relação entre a massa de grãos ou da parte aérea e a quantidade de $\mathrm{N}$ aplicado. A EUN é determinada pela eficiência na absorção de nitrogênio (EAN), definida como a capacidade da planta em absorver $\mathrm{N}$, e pela eficiência na utilização de nitrogênio
(EUtN), considerada como a capacidade para produzir grãos (final do ciclo) ou matéria seca (início do ciclo) com o $\mathrm{N}$ absorvido. Dessa forma, valores superiores de EUN podem ser obtidos pelo aumento em EAN ou em EUtN.

Entre as propostas para melhorar a EUtN em milho, destacam-se o aumento da atividade enzimática nas raízes (Chevalier \& Schrader, 1977) e o aumento da quantidade de matéria seca produzida por unidade de N (Maranville et al., 1980). No caso da EAN, ganhos na absorção de $\mathrm{N}$ podem ser alcançados pelo aumento 
em extensão do sistema radicular, principalmente em ambientes com baixa disponibilidade de N (Garnett et al., 2009).

Le Gouis et al. (2000) verificaram que a variabilidade genética detectada em trigo quanto a EUN, em condições limitantes de N, ocorre em função das diferenças em EAN. No entanto, Gallais \& Hirel (2004) observaram que, nessas condições, a EUtN é o componente mais importante para a EUN, em milho. Nesse sentido, Oliveira (2009) constatou ganhos indiretos de $24,93 \%$ para a EUN, em baixa disponibilidade de $\mathrm{N}$, por meio da atividade da enzima glutamina sintetase, o que confirma a importância da EUtN para a EUN. Entretanto, em razão da grande quantidade de genótipos obtida por ciclo nos programas de melhoramento, é pouco viável discriminá-los com base na atividade enzimática, uma vez que a metodologia necessária é morosa e custosa. Assim, identificar caracteres de mais fácil mensuração é fundamental para a seleção de genótipos superiores para condições de estresse abiótico, pois acelera o processo de seleção e permite descartar de imediato genótipos menos promissores (Machado et al., 2004). Para isso, torna-se necessário o conhecimento da relação entre os caracteres envolvidos na eficiência nutricional, e que estes sejam passíveis de mensuração em estádios precoces de desenvolvimento.

Segundo Maia et al. (2011), a massa de matéria seca da parte aérea e o comprimento de raízes possibilitam a seleção de genótipos de forma rápida e a baixo custo. Contudo, ainda são poucos os estudos sobre a influência desses caracteres na EUN. O conhecimento dessas informações e da herança genética em condições contrastantes de disponibilidade de $\mathrm{N}$ é crucial para orientar o melhorista quanto ao método mais adequado de melhoramento a ser utilizado. Neste sentido, parâmetros genéticos, como capacidade geral (CGC) e específica (CEC) de combinação, são importantes para inferir sobre o controle genético de caracteres de interesse e sobre as possibilidades de melhoramento, bem como para indicação de novas cultivares de milho (Betrán et al., 2003).

Os objetivos deste trabalho foram determinar o controle genético de EUN, identificar a importância de EAN e de EUtN na sua composição, e quantificar a relação entre produção de matéria seca da parte aérea (MPS) e do sistema radicular com a EUN e com seus componentes.

\section{Material e Métodos}

O experimento foi conduzido em casa de vegetação, no Campo Experimental Prof. Diogo Alves de Mello, do Departamento de Fitotecnia, da Universidade Federal de Viçosa $\left(20^{\circ} 45^{\prime} 14^{\prime \prime} \mathrm{S}, 42^{\circ} 52^{\prime} 53^{\prime \prime} \mathrm{W}\right)$, no ano agrícola de 2010/2011. Foram utilizados dois grupos de linhagens de milho (Zea mays L.), provenientes do banco de germoplasma do Programa Milho da UFV: grupo I (GI), composto de sete linhagens $(\mathrm{p}=1,2, \ldots, 7)$ contrastantes quanto à eficiência no uso de fósforo (EUP); e grupo II (GII), composto de sete linhagens ( $\mathrm{p}^{\prime}=1$ ', 2', ..., 7') contrastantes quanto a EUN. Foram obtidas 41 combinações híbridas, por meio da condução de um fatorial interpopulacional desbalanceado, com uso dos dois grupos de linhagens. Os cruzamentos foram realizados de forma recíproca, tendo-se misturado as sementes da mesma combinação, e, portanto, desconsiderado a herança extracromossômica.

As 41 combinações híbridas foram avaliadas em duas disponibilidades de N: baixa e alta. Utilizou-se o delineamento experimental de blocos ao acaso com duas repetições, em arranjo fatorial simples (combinação híbrida $\mathrm{x}$ disponibilidade de $\mathrm{N}$ ). As sementes foram germinadas em bandejas com células individuais. Após a emergência, transplantou-se uma plântula de cada combinação híbrida para vasos cilíndricos de PVC com 4,0 dm dm $^{3} 10 \mathrm{~cm}$ de diâmetro x $50 \mathrm{~cm}$ de altura), o que constituiu uma parcela. $\mathrm{O}$ substrato utilizado foi preparado pela mistura de areia lavada em água deionizada e vermiculita, na proporção de 1:1, conforme Walk et al. (2006).

Foi utilizada a solução nutritiva descrita por Chun et al. (2005) contendo, para alto $\mathrm{N}: 2,0 \mathrm{mmol} \mathrm{L}^{-1}$ de $\mathrm{Ca}\left(\mathrm{NO}_{3}\right)_{2} ; 0,75 \mathrm{mmol} \mathrm{L}^{-1}$ de $\mathrm{K}_{2} \mathrm{SO}_{4} ; 0,65 \mathrm{mmol} \mathrm{L}^{-1}$ de $\mathrm{MgSO}_{4} ; 0,1 \mathrm{mmol} \mathrm{L}^{-1}$ de $\mathrm{KCl} ; 0,25 \mathrm{mmol} \mathrm{L}^{-1}$ de $\mathrm{KH}_{2} \mathrm{PO}_{4}$; $1 \times 10^{-3} \mathrm{mmol} \mathrm{L}^{-1}$ de $\mathrm{H}_{3} \mathrm{BO}_{3} ; 1 \times 10^{-3} \mathrm{mmol} \mathrm{L}^{-1}$ de $\mathrm{MnSO}_{4}$; $1 \times 10^{-4} \mathrm{mmol} \mathrm{L}^{-1}$ de $\mathrm{CuSO}_{4} ; 1 \times 10^{-3} \mathrm{mmol} \mathrm{L}^{-1}$ de $\mathrm{ZnSO}_{4}$; $5 \times 10^{-6} \mathrm{mmol} \mathrm{L}^{-1}$ de $\left(\mathrm{NH}_{4}\right)_{6} \mathrm{Mo}_{7} \mathrm{O}_{24}$; e $0,1 \mathrm{mmol} \mathrm{L}^{-1}$ de Fe-EDTA. Para baixo N, a solução continha: 0,2 mmol L-1 de $\mathrm{Ca}\left(\mathrm{NO}_{3}\right)_{2}$, ou seja, concentração dez vezes menor que a do alto $\mathrm{N}$. O $\mathrm{Ca}^{2+}$ foi compensado pelo $\mathrm{CaCl}_{2}$. A solução foi fornecida a cada dois dias, a partir do sétimo dia após a emergência das plântulas.

As plantas foram colhidas no estádio vegetativo V6. A parte aérea das plantas foi separada do sistema radicular e acondicionada em sacos de papel. Posteriormente, procedeu-se à secagem em estufa de 
circulação forçada de ar a $60^{\circ} \mathrm{C}$ por 72 horas, até atingir massa constante.

Todo o sistema radicular foi avaliado pela análise de imagens, com uso do programa WinRHIZO Pro 2009c (Regent Instruments Inc., Quebec, Canadá) acoplado a escâner Epson Expression 10.000 XL (Epson Brasil, São Paulo, SP) equipado com luz adicional, com definição de 400 dpi, conforme Bouma et al. (2000). Para facilitar a caracterização das combinações híbridas quanto ao sistema radicular, as dez classes de diâmetro fornecidas pelo programa foram simplificadas em apenas duas, de acordo com Trachsel et al. (2009). Portanto, para o comprimento das raízes laterais $\left(\mathrm{CR}_{\mathrm{LAT}}\right)$, foram considerados fragmentos com diâmetro menor ou igual a $0,5 \mathrm{~mm}$, e, para o comprimento das raízes axiais $\left(\mathrm{CR}_{\mathrm{AXI}}\right)$, fragmentos com diâmetro maior que $0,5 \mathrm{~mm}$.

Para a quantificação de N, extraíram-se amostras de $0,2 \mathrm{~g}$ da MPS por planta. Em seguida, foram feitas a digestão, a destilação e a titulação, conforme Bremner \& Mulvaney (1982). Após isso, calculou-se o teor de $\mathrm{N}$ em cada amostra (concentração de $\mathrm{N}$ por amostra). $\mathrm{O}$ conteúdo de $\mathrm{N}$ foi estimado ao se extrapolar o teor por unidade de MPS. A partir dos conteúdos, calculou-se a EAN, a EUtN e a EUN, de acordo com Moll et al. (1982), como: $\mathrm{EAN}=\mathrm{N}($ absorvido $) / \mathrm{N}$ (aplicado) $\left(\mathrm{mg} \mathrm{mg}{ }^{-1}\right), \mathrm{EUtN}=$ MPS/N(absorvido) $\left(\mathrm{mg} \mathrm{mg}^{-1}\right) \mathrm{e}$ $\mathrm{EUN}=\mathrm{EAN} \times \operatorname{EUtN}\left(\mathrm{mg} \mathrm{mg}^{-1}\right)$.

Para a obtenção dos componentes de variância e das estimativas dos parâmetros genéticos, os dados foram submetidos às análises por meio do método da máxima verossimilhança restrita/melhor preditor linear não viesado (REML/Blup), conforme Resende (2002). Inicialmente, procederam-se às análises de deviance individuais, para cada disponibilidade de $\mathrm{N}$, tendo-se considerado o seguinte modelo: $\mathrm{y}=\mathrm{Xr}+$ $\mathrm{Zm}+\mathrm{Wf}+\mathrm{Tc}+\mathrm{e}$, em que: y é o vetor das médias fenotípicas das combinações híbridas; $r$ é o vetor dos efeitos de repetição (assumidos como fixos) somados à média geral; $\mathrm{m}$ é o vetor dos efeitos de genitores do GII (assumidos como aleatórios), em que $\mathrm{m} \sim \mathrm{N}(0, \mathrm{M})$, sendo $M=I \sigma_{\mathrm{m}}^{2}$; f é o vetor dos efeitos de genitores do GI (assumidos como aleatórios), em que $\mathrm{f} \sim \mathrm{N}(0, \mathrm{~F})$, sendo $\mathrm{F}=\mathrm{I} \sigma_{\mathrm{f}}^{2}$, c é o vetor dos efeitos da capacidade específica de combinação do GI com o GII (assumidos como aleatórios), em que $\mathrm{c} \sim \mathrm{N}(0, \mathrm{C})$, sendo $\mathrm{C}=\mathrm{I}_{\mathrm{c}}^{2}$; e e é o vetor de erros, em que, e $\sim N(0, R)$, sendo $R=I \sigma_{\mathrm{e}}^{2}$.
$\mathrm{X}, \mathrm{Z}, \mathrm{W}$ e T são matrizes de incidência que relacionam, respectivamente, os efeitos de $\mathrm{r}, \mathrm{m}, \mathrm{f}, \mathrm{e} \mathrm{c}$ ao vetor $\mathrm{y}$.

As equações de modelos mistos para a predição de $r$, $\mathrm{m}, \mathrm{f}$ e c equivalem a:

$\left[\begin{array}{cccc}X^{\prime} X & X^{\prime} Z & X^{\prime} W & X^{\prime} T \\ Z^{\prime} X & Z^{\prime} Z+\lambda_{1} & Z^{\prime} W & Z^{\prime} T \\ W^{\prime} X & W^{\prime} Z & W^{\prime} W+\lambda_{2} & W^{\prime} T \\ T^{\prime} X & T^{\prime} Z & T^{\prime} W & T^{\prime} T+\lambda_{3}\end{array}\right]\left[\begin{array}{c}r \\ m \\ f \\ c\end{array}\right]=\left[\begin{array}{c}X^{\prime} y \\ Z^{\prime} y \\ W^{\prime} y \\ T^{\prime} y\end{array}\right]$

em que: $\lambda_{1}=\left(1-h_{m}^{2}-h_{f}^{2}-h_{c}^{2}\right) / h_{m}^{2}, \lambda_{2}=\left(1-h_{m}^{2}-h_{f}^{2}-h_{c}^{2}\right) / h_{f}^{2} e$ $\lambda_{3}=\left(1-h_{m}^{2}-h_{f}^{2}-h_{c}^{2}\right) / h_{c}^{2}$, em que $h_{m}^{2}$ é a herdabilidade no sentido restrito dos genitores do GI; $h_{\mathrm{f}}^{2}$ é a herdabilidade no sentido restrito dos genitores do GII; e $h_{c}^{2}$ é o coeficiente de determinação dos efeitos da capacidade específica de combinação do GI com o GII.

Posteriormente, foram realizadas as análises de deviance conjuntas, para as duas disponibilidades de $\mathrm{N}$, ao se considerar o modelo simplificado: $\mathrm{y}=\mathrm{Xr}+\mathrm{Zg}$ $+\mathrm{Wi}+\mathrm{e}$, em que: y é o vetor das médias fenotípicas das combinações híbridas; $r$ é o vetor dos efeitos de repetição dentro das disponibilidades de $\mathrm{N}$ (assumidos como fixos) somados à média geral; g é o vetor dos efeitos genotípicos (assumidos como aleatórios), em que $\mathrm{g} \sim \mathrm{N}(0, \mathrm{~g})$, sendo $\mathrm{G}=\mathrm{I} \sigma_{\mathrm{g}}^{2}$; i é o vetor da interação combinação híbrida $\mathrm{x}$ disponibilidade de $\mathrm{N}$ (assumidos como aleatórios), em que $\mathrm{i} \sim \mathrm{N}\left(0, \mathrm{I}_{\mathrm{ga}}\right)$, sendo $\mathrm{I}_{\mathrm{ga}}=\mathrm{I} \sigma_{\mathrm{ga}}^{2}$; e e é o vetor de erros, em que, e $\sim N(0, R)$, sendo $R=I \sigma_{e}^{2}$. $\mathrm{X}, \mathrm{Z}$ e W são matrizes de incidência que relacionam, respectivamente, os efeitos de $\mathrm{r}$, g e i ao vetor $\mathrm{y}$.

As equações de modelo misto para a predição de $r$, $\mathrm{g}$ e i equivalem a:

$$
\left[\begin{array}{ccc}
X^{\prime} X & X^{\prime} Z & X^{\prime} W \\
Z^{\prime} X & Z^{\prime} Z+\lambda_{1} & Z^{\prime} W \\
W^{\prime} X & W^{\prime} Z & W^{\prime} W+\lambda_{2}
\end{array}\right]\left[\begin{array}{c}
r \\
g \\
i
\end{array}\right]=\left[\begin{array}{c}
X^{\prime} y \\
Z^{\prime} Y \\
W^{\prime} y
\end{array}\right]
$$

em que: $\lambda_{1}=\left(1-h_{\mathrm{g}}^{2}-\mathrm{h}_{\mathrm{i}}^{2}\right) / \mathrm{h}_{\mathrm{g}}^{2}$ e $\lambda_{2}=\left(1-\mathrm{h}_{\mathrm{g}}^{2}-\mathrm{h}_{\mathrm{i}}^{2}\right) / \mathrm{h}_{\mathrm{i}}^{2}$, em que $\mathrm{h}_{\mathrm{g}}^{2}$ é a herdabilidade no sentido amplo das combinações híbridas e $h_{i}^{2}$ é o coeficiente de determinação da interação combinação híbrida $\mathrm{x}$ disponibilidade de $\mathrm{N}$.

Para a resolução das equações de modelos mistos e a obtenção dos valores genéticos, os componentes de variância genéticos e não genéticos foram assumidos desconhecidos. Esses valores foram estimados pelo método REML, com uso do pacote estatístico Selegen-REML/Blup (Resende, 2007). 
Para conhecer a relação entre as respostas das combinações híbridas em baixo e alto $\mathrm{N}\left(\mathrm{r}_{\mathrm{AN} \times \mathrm{BN}}\right)$, estimou-se a correlação entre o ranking dessas combinações, ao se considerar o desempenho do mesmo caráter nas duas disponibilidades de N. Para isso, utilizou-se o coeficiente de correlação de postos de Spearman $(\rho)$, por meio da expressão $\rho=\left(1-6 \sum \mathrm{d}_{\mathrm{i}}^{2}\right) /$ $n\left(n^{2}-1\right)$, em que: $d_{i}^{2}$ é a diferença entre o posto de cada valor correspondente em baixo e alto $\mathrm{N}$; e n é o número de pares de valores.

\section{Resultados e Discussão}

Em baixo N, os genitores do GI não apresentaram diferença significativa para o efeito de CGC quanto aos caracteres avaliados (Tabela 1). No entanto, os genitores do GII diferiram para a maioria dos caracteres, com exceção da EUtN. Essas diferenças indicam a existência de variabilidade genética aditiva e que os genitores contribuem de maneira diferenciada na transmissão de alelos que controlam a EUN. Esses resultados já eram esperados, pois foram identificados, em experimento preliminar, genitores contrastantes quanto à EUP, para compor o GI, e à EUN, para constituir o GII, o que comprova que a identificação desses genitores foi eficiente. No entanto, não foi constatada diferença significativa para o efeito de CEC para os caracteres avaliados. Isso mostra que, em baixo $\mathrm{N}$, as combinações híbridas não apresentaram, em geral, desvios significativos em relação ao comportamento médio dos genitores. Em alto N, os genitores do GII apresentaram diferença significativa para o efeito de CGC, em todos os caracteres avaliados.

As diferenças significativas constatadas para a EUN podem ser explicadas, em baixo $\mathrm{N}$, pela variabilidade genética aditiva detectada para a EAN, e, em alto N, pela variabilidade existente tanto para a EAN quanto para a EUtN (Tabela 1). Contudo, para ambas as disponibilidades de $\mathrm{N}$, os efeitos genéticos aditivos apresentaram maior importância para os caracteres associados à EUN. Below et al. (1997) verificaram diferenças significativas da CGC e da CEC para os caracteres associados à EUN, o que foi atribuído à superioridade dos valores da CGC e à maior importância dos efeitos aditivos para os componentes da EUN.

Foram observadas pequenas diferenças entre as magnitudes das estimativas de herdabilidade no sentido amplo e restrito, especialmente em alto N. Assim, a variância genotípica é explicada, principalmente, pela variância aditiva, o que foi comprovado pelos baixos valores da razão $\hat{\sigma}_{\mathrm{d}}^{2} / \hat{\sigma}_{\mathrm{a}}^{2}$, na maioria dos caracteres. Dessa forma, fica evidente que os efeitos não aditivos têm menor importância para os componentes da EUN, como relatado por Souza et al. (2008) e Worku et al. (2008). Portanto, a seleção pode ser realizada pelo desempenho individual das linhagens, uma vez que as combinações híbridas obtidas entre os seus cruzamentos também serão superiores em relação à EUN.

Para a maioria dos caracteres, as estimativas de média e herdabilidade foram, em geral, menores em baixo $\mathrm{N}$.

Tabela 1. Teste da razão de verossimilhança para os efeitos de capacidade geral de combinação (CGC) dos grupos I (GI) e II (GII) e dos efeitos de capacidade especifica de combinação (CEC) entre os dois grupos das análises de deviance individuais, estimativas das herdabilidades nos sentidos restrito $\left(\mathrm{h}_{\mathrm{a}}^{2}\right)$ e amplo $\left(\mathrm{h}_{\mathrm{g}}^{2}\right)$, razão entre a variância dos desvios de dominância e a variância aditiva $\left(\hat{\sigma}_{\mathrm{d}}^{2} / \hat{\sigma}_{\mathrm{a}}^{2}\right)$, médias e coeficientes de variação $(\mathrm{CV})$ da massa de matéria seca da parte aérea (MPS), comprimento de raízes laterais $\left(\mathrm{CR}_{\mathrm{LAT}}\right)$ e axiais $\left(\mathrm{CR}_{\mathrm{AXI}}\right)$, eficiências na absorção (EAN), na utilização (EUtN) e no uso de $\mathrm{N}$ (EUN), em baixo (BN) e alto $\mathrm{N}(\mathrm{AN})$, em 41 combinações híbridas de milho.

\begin{tabular}{|c|c|c|c|c|c|c|c|c|c|c|c|c|}
\hline \multirow[t]{2}{*}{ Efeito } & \multicolumn{2}{|c|}{ MPS } & \multicolumn{2}{|c|}{$\mathrm{CR}_{\mathrm{LAT}}$} & \multicolumn{2}{|c|}{$\mathrm{CR}_{\mathrm{AXI}}$} & \multicolumn{2}{|c|}{ EAN } & \multicolumn{2}{|c|}{ EUtN } & \multicolumn{2}{|c|}{ EUN } \\
\hline & $\mathrm{BN}$ & AN & $\mathrm{BN}$ & AN & $\mathrm{BN}$ & AN & $\mathrm{BN}$ & AN & $\mathrm{BN}$ & AN & $\mathrm{BN}$ & AN \\
\hline$\overline{C G C}(\mathrm{GI})^{(1)}$ & $0^{\mathrm{ns}}$ & $3,81 *$ & $0,32^{\text {ns }}$ & $1,19^{\mathrm{ns}}$ & $1,89^{\text {ns }}$ & $3,86^{*}$ & $0,05^{\text {ns }}$ & $4,49 *$ & $1,67^{\mathrm{ns}}$ & $0^{\text {ns }}$ & $0^{\mathrm{ns}}$ & $3,40^{\mathrm{ns}}$ \\
\hline $\mathrm{CGC}(\mathrm{GII})^{(1)}$ & $3,97 *$ & $14,19 * *$ & $3,84 *$ & $7,18 * *$ & $6,9 * *$ & $7,71 * *$ & $11,53 * *$ & $13,20 * *$ & $0,03^{\text {ns }}$ & $9,21 * *$ & $3,96^{*}$ & $14,18 * *$ \\
\hline CEC GI x GII ${ }^{(1)}$ & $1,66^{\mathrm{ns}}$ & $0,14^{\mathrm{ns}}$ & $1,21^{\mathrm{ns}}$ & $0,13^{\text {ns }}$ & $1,58^{\mathrm{ns}}$ & $2,68^{\mathrm{ns}}$ & $0,13^{\text {ns }}$ & $0,23^{\mathrm{ns}}$ & Ons & $2,70^{\mathrm{ns}}$ & $1,67^{\mathrm{ns}}$ & $0,15^{\mathrm{ns}}$ \\
\hline $\mathrm{h}_{\mathrm{a}}^{2}$ & 0,13 & 0,43 & 0,14 & 0,26 & 0,24 & 0,27 & 0,29 & 0,42 & 0,09 & 0,22 & 0,13 & 0,43 \\
\hline $\mathrm{h}_{\mathrm{g}}^{2}$ & 0,32 & 0,47 & 0,31 & 0,31 & 0,41 & 0,59 & 0,34 & 0,47 & 0,10 & 0,44 & 0,32 & 0,47 \\
\hline$\hat{\sigma}_{\mathrm{d}}^{2} / \hat{\sigma}_{\mathrm{a}}^{2}$ & 1,43 & 0,09 & 1,19 & 0,18 & 0,68 & 1,18 & 0,15 & 0,12 & 0,14 & 0,95 & 1,43 & 0,09 \\
\hline Média & 0,45 & 0,92 & 19,74 & 21,92 & 8,02 & 8,80 & 0,33 & 0,10 & 35,22 & 24,59 & 11,51 & 2,34 \\
\hline CV $(\%)$ & 19,54 & 22,31 & 24,63 & 26,32 & 23,67 & 20,96 & 20,75 & 24,07 & 13,02 & 7,62 & 19,54 & 22,31 \\
\hline
\end{tabular}

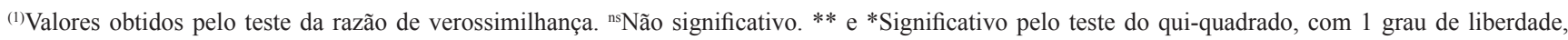
a 1 e $5 \%$ de probabilidade, respectivamente. 
Segundo Coque \& Gallais (2006), em condições de estresse abiótico, ocorre redução da variabilidade genética e, consequentemente, da herdabilidade, pois os genótipos tendem a apresentar desempenhos similares, o que dificulta a seleção. As exceções foram para a EUN e os seus componentes, já que a maioria desses caracteres é mensurada em função da MPS, a qual não foi reduzida na mesma proporção que a disponibilidade de $\mathrm{N}$.

Observou-se, pelas análises de deviance conjuntas, que as disponibilidades de $\mathrm{N}$ foram suficientemente contrastantes para discriminar o desempenho das combinações híbridas em relação à maioria dos caracteres, exceto para os comprimentos de raízes (Tabela 2). Além disso, verificou-se que o efeito de combinações híbridas apresentou diferença não significativa para os caracteres avaliados. A variância genética detectada na análise conjunta corresponde à média das variâncias nos dois ambientes avaliados, subtraída a interação. Por essa razão, quando se trabalha com ambientes contrastantes é comum que a variabilidade genética observada em cada ambiente seja "anulada" pela interação na análise conjunta, pois podem apresentar sentidos contrários de expressão gênica. Contudo, as combinações híbridas responderam diferentemente em baixo e alto $\mathrm{N}$, o que foi evidenciado pelos efeitos significativos da interação combinação híbrida x disponibilidade de N, exceto para a EUtN. Isso indica que a seleção deveria ser realizada em ambientes específicos e não em função do comportamento médio, pois os alelos que controlam a expressão de determinado caráter, em baixa suplementação nutricional, são, pelo menos parcialmente, diferentes dos alelos que controlam esse mesmo caráter em condição ideal de suplementação (Souza et al., 2008). As correlações entre os desempenhos das combinações híbridas em baixo e alto $\mathrm{N}\left(\mathrm{r}_{\mathrm{AN} \times \mathrm{BN}}\right)$ apresentaram estimativas de média a alta magnitude $(>0,70)$ para todos os caracteres avaliados, com exceção da EUtN. Isso é indicativo de interação do tipo simples (Resende, 2002), pois reflete a consistência da superioridade dos genótipos mesmo ao se mudar a disponibilidade de $\mathrm{N}$, ou seja, as combinações híbridas com desempenho superior em baixo $\mathrm{N}$ também são as melhores em alto N. Desse modo, híbridos de milho podem ser avaliados em um único ambiente (baixo ou alto $\mathrm{N}$ ), e as decisões nele tomadas poderão ser válidas para outro (Falconer, 1981), o que economizaria recursos, tempo e mão de obra.

Os coeficientes de variação, tanto das análises de deviance individuais quanto das conjuntas, foram relativamente altos para alguns caracteres. No entanto, estão no intervalo observado para estudos dessa natureza, ou seja, em estresse abiótico (Bänziger \& Lafitte, 1997; Machado et al., 2004), o que indica boa precisão experimental e alta confiabilidade das estimativas.

Os coeficientes de correlação genotípica $\left(\mathrm{r}_{\mathrm{g}}\right)$ apresentaram, em sua maioria e independentemente da disponibilidade de $\mathrm{N}$, estimativas significativas de média a alta magnitude (Tabela 3). Incrementos em MPS proporcionam aumentos no sistema radicular, o

Tabela 2. Valores de F calculado para o efeito de disponibilidade de $\mathrm{N}$ e do teste de razão de verossimilhança para os efeitos de combinação híbrida e da interação combinação híbrida x disponibilidade de $\mathrm{N}$ das análises de deviance conjuntas, estimativas das herdabilidades no sentido amplo $\left(\mathrm{h}_{\mathrm{g}}^{2}\right)$, correlações entre os desempenhos dos híbridos nas duas disponibilidades de $\mathrm{N}$ ( $\mathrm{r}_{\mathrm{AN} \text { x BN}}$ ), médias e coeficientes de variação $(\mathrm{CV})$ da massa de matéria seca da parte aérea seca (MPS), comprimento de raízes laterais $\left(\mathrm{CR}_{\mathrm{LAT}}\right)$ e axiais $\left(\mathrm{CR}_{\mathrm{AxI}}\right)$, eficiências na absorção (EAN), na utilização (EUtN) e no uso de $\mathrm{N}$ (EUN), em baixo e alto $\mathrm{N}$, em 41 combinações híbridas de milho.

\begin{tabular}{lllllll}
\hline Efeito & MPS & $\mathrm{CR}_{\mathrm{LAT}}$ & $\mathrm{CR}_{\mathrm{AXI}}$ & $\mathrm{EAN}$ & $\mathrm{EUtN}$ & EUN \\
\hline Disponibilidade de $\mathrm{N}(\mathrm{N})^{(1)}$ & $9,00^{* *}$ & $0,19^{\text {ns }}$ & $0,18^{\text {ns }}$ & $19,20^{* *}$ & $8,94^{* *}$ & $31,69^{* *}$ \\
Combinação híbrida $(\mathrm{CH})^{(2)}$ & $0,98^{\text {ns }}$ & $0,01^{\text {ns }}$ & $0,03^{\text {ns }}$ & $0,57^{\text {ns }}$ & $0,88^{\text {ns }}$ & $0,64^{\text {ns }}$ \\
$\mathrm{CH} \mathrm{x} \mathrm{N}^{(2)}$ & $21,8^{* *}$ & $24,37^{* *}$ & $42,86^{* *}$ & $12,9^{* *}$ & $0,43^{\text {ns }}$ & $11,86^{* *}$ \\
$\mathrm{~h}_{\mathrm{g}}^{2}$ & 0,31 & 0,36 & 0,49 & 0,23 & 0,01 & 0,22 \\
$\mathrm{r}_{\mathrm{AN} \text { BN }}$ & 0,77 & 0,99 & 0,97 & 0,75 & 0,10 & 0,72 \\
\hline Média & 0,69 & 21,00 & 8,44 & 0,22 & 29,88 & 6,95 \\
$\mathrm{CV}(\%)$ & 22,71 & 23,89 & 21,98 & 23,86 & 11,90 & 23,44 \\
\hline
\end{tabular}

${ }^{(1)}$ Valores de $\mathrm{F}$ calculado, testados com 1 e 40 graus de liberdade. ns Não significativo. **Significativo pelo teste $\mathrm{F}$, a $1 \%$ de probabilidade. (2) Valores

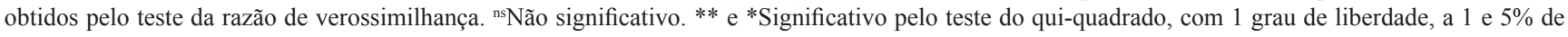
probabilidade, respectivamente. 
que foi comprovado pelas correlações positivas e de altas magnitudes $(>0,80)$ entre esses caracteres. A alta correlação detectada entre comprimentos de raízes axiais e laterais, em baixo e alto $\mathrm{N}$, indica a existência de dependência hierárquica, isto é, à medida que aumenta o comprimento das matrizes axiais aumenta o número de raízes laterais com maior área para captação de N. Consequentemente, maiores comprimentos de raízes devem causar aumentos na EAN, tanto em baixo como em alto $\mathrm{N}$.

Observou-se correlação negativa e significativa entre a EAN e a EUtN nas duas condições de disponibilidade de N. As plantas de milho nos estádios iniciais de desenvolvimento provavelmente absorvem melhor os nutrientes que utilizam, o que torna a EAN o componente mais importante nesses estádios. Entretanto, com o decorrer do desenvolvimento, o componente da utilização tem sua importância aumentada em detrimento da absorção. Assim, tanto em estádios iniciais como nos mais avançados, os componentes da eficiência no uso de nutrientes não apresentam proporcionalidade. Gallais \& Hirel (2004) observaram resultados semelhantes em milho e propuseram três hipóteses para explicar esse fenômeno: as atividades dos transportadores de $\mathrm{N}$ e das enzimas envolvidas na assimilação desse nutriente ocorrem em proporção diferente; há degradação de proteínas foliares (em específico, rubisco) que contribuem adicionalmente para a produção; e a utilização do $\mathrm{N}$ é mais pronunciada onde a absorção é reduzida ou interrompida.

Para Le Gouis et al. (2000), o componente mais importante para a EUN em baixo $\mathrm{N}$ é a EAN. Já Gallais \& Hirel (2004), reportaram que, em alto N, a variabilidade genética para a EUN ocorre em função das diferenças na EAN, enquanto, em baixo N, é função da EUtN. Contudo, no presente trabalho, verificou-se, por meio de correlações de elevada magnitude, que, independentemente da disponibilidade de $\mathrm{N}$, as diferenças genéticas quanto à EUN foram decorrentes da EAN (Tabela 1). A ausência de correlação entre a EUtN e a EUN é indicativo de redução da diversidade alélica em relação à EUtN, uma vez que as cultivares modernas, em sua quase totalidade, são selecionadas para responsividade em condições de alta disponibilidade nutricional.

Apesar de ter sido constatada correlação positiva e significativa entre o comprimento das raízes e a EUN, é provável que não seja possível selecionar genótipos mais eficientes com base nestes caracteres radiculares. Lima (2010) e Pereira (2011), por meio da análise de trilha, observaram que a alta correlação entre os caracteres supracitados pode ser atribuída ao elevado efeito indireto via MPS, seja em baixo ou alto N. Para estes autores, caracteres como a MPS e a EAN podem proporcionar maior impacto nos ganhos genéticos. No presente trabalho, verificou-se correlação linear e positiva entre a MPS e a EUN, nas duas condições de disponibilidade de N. Diante desses resultados e das diferenças significativas da EAN constatadas nas duas disponibilidades de N, plotou-se, em um plano cartesiano, as estimativas de CGC dos genitores quanto à MPS e à EAN (Figura 1). No quadrante I (QI), encontram-se os genitores com valores positivos dos caracteres tanto em baixo como em alto N. Analogamente, no QIII, estão os genitores que apresentaram valores negativos em ambas as disponibilidades de N; no QII, os genitores que apresentaram valores negativos em baixo $\mathrm{N}$, mas positivos em alto N; e, no QIV, o contrário. Assim, pôde-se verificar que o desempenho das linhagens genitoras, baseado nas estimativas de CGC da MPS, coincidiu em 13 dos 14 genitores avaliados, com as estimativas de CGC da EAN. A única exceção foi o

Tabela 3. Estimativas de correlações genotípicas entre massa de matéria seca de parte aérea (MPS), comprimento de raízes laterais $\left(\mathrm{CR}_{\mathrm{LAT}}\right)$ e axiais $\left(\mathrm{CR}_{\mathrm{AXI}}\right)$, eficiências na absorção (EAN), na utilização (EUtN) e no uso de $\mathrm{N}$ (EUN), em baixo (acima da diagonal) e alto $\mathrm{N}$ (abaixo da diagonal), em 41 combinações híbridas de milho.

\begin{tabular}{|c|c|c|c|c|c|c|}
\hline Caráter & MPS & $\mathrm{CR}_{\mathrm{LAT}}$ & $\mathrm{CR}_{\mathrm{AXI}}$ & EAN & EUtN & EUN \\
\hline MPS & & $0,88 * *$ & $0,86^{* *}$ & $0,85^{* *}$ & $0,18^{\text {ns }}$ & $1,00 * *$ \\
\hline $\mathrm{CR}_{\mathrm{LAT}}$ & $0,98 * *$ & & $0,70 * *$ & $0,85^{* *}$ & $0,03^{\mathrm{ns}}$ & $0,88 * *$ \\
\hline $\mathrm{CR}_{\mathrm{AXI}}$ & $0,83 * *$ & $0,91 * *$ & & $0,92 * *$ & $-0,23^{\mathrm{ns}}$ & $0,86^{* *}$ \\
\hline EAN & $0,99 * *$ & $0,98 * *$ & $0,86^{* *}$ & & $-0,35^{*}$ & $0,85 * *$ \\
\hline EUtN & $-0,56 * *$ & $-0,62 * *$ & $-0,74 * *$ & $-0,64 * *$ & & $-0,18^{\mathrm{ns}}$ \\
\hline EUN & $1,00 * *$ & $0,98 * *$ & $0,83 * *$ & $0,99 * *$ & $-0,26^{\mathrm{ns}}$ & \\
\hline
\end{tabular}

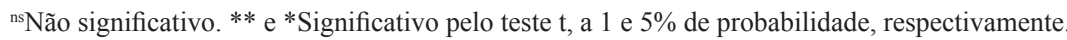


genitor 2, que teve a estimativa de CGC da MPS plotada no QI e a da EAN no QIV. Esses resultados estão de acordo com os obtidos por Pereira (2011), que identificou a MPS como caráter fundamental na seleção precoce e indireta de genótipos de milho com alta EUN.

A MPS pode consistir em um indicador de produtividade, se o índice de colheita (IC) - tido como a fração dos grãos produzidos em relação à massa de matéria seca da planta - for levado em consideração. Em milho, esses valores estão estagnados próximos de 0,60 há décadas (Raun et al., 1989). Nesse sentido, valores adicionais em MPS podem implicar em aumento de produtividade, o que indica que esse caráter deve ser utilizado em programas de melhoramento de plantas para condições de estresse abiótico, com redução do tempo e do trabalho demandado.

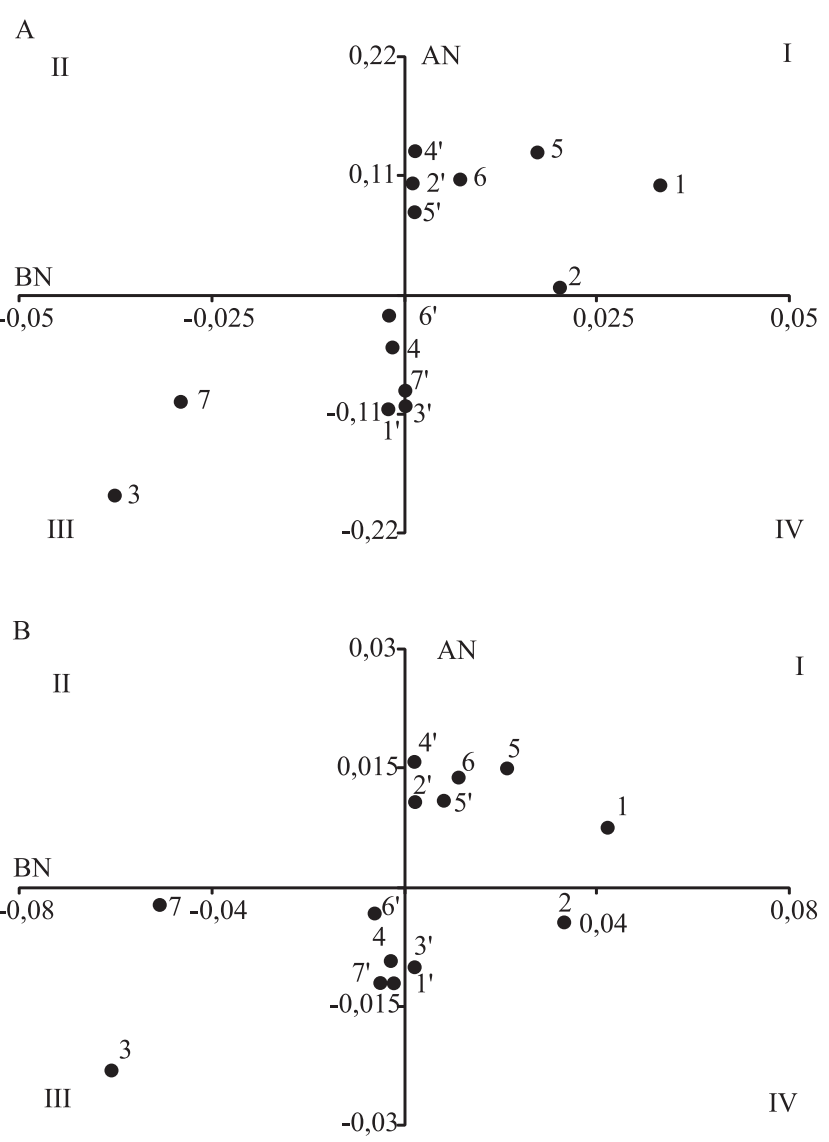

Figura 1. Capacidade geral de combinação das 14 linhagens genitoras de milho quanto à massa de matéria seca da parte aérea (A) e a eficiência na absorção de $\mathrm{N}$ (B) em baixo (eixo das abscissas) e alto $\mathrm{N}$ (eixo das ordenadas).

\section{Conclusões}

1. Independentemente da disponibilidade de $\mathrm{N}$, a eficiência na absorção é o componente mais importante da eficiência no uso de N.

2. A forte associação entre massa de matéria seca da parte aérea e eficiência na absorção permite a seleção baseada neste primeiro caráter, para obtenção de genótipos de milho mais eficientes no uso de $\mathrm{N}$ em estádio precoce de desenvolvimento.

3. Os efeitos genéticos aditivos têm maior importância para os caracteres associados à eficiência no uso de $\mathrm{N}$, de modo que a seleção pode ser realizada pelo desempenho individual de linhagens.

4. A interação combinação híbrida $\mathrm{x}$ disponibilidade de $\mathrm{N}$ é predominantemente do tipo simples e, portanto, a seleção pode ser realizada em uma única disponibilidade de $\mathrm{N}$.

\section{Referências}

BÄNZIGER, M.; LAFITTE, H.R. Efficiency of secondary traits for improving maize for low-nitrogen target environments. Crop Science, v.37, p.1110-1117, 1997.

BELOW, F.E.; BRANDAU, P.S.; LAMBERT, R.J.; TEYKER, R.H. Combining ability for nitrogen use in maize. In: EDMEADES, G.O.; BÄNZIGER, M.; MICKELSON, H.R.; PEÑA-VALDIVA, C.B. Developing drought and low n-tolerant maize. Mexico, D.F.: CIMMYT, 1997. p.316-319.

BETRÁN, F.J.; BECK, D.; BÄNZIGER, M.; EDMEADES, G.O. Genetic analysis of inbred and hybrid grain yield under stress and nonstress environments in tropical maize. Crop Science, v.43, p.807-817, 2003.

BOUMA, T.J.; NIELSEN, K.L.; KOUTSTAAL, B. Sample preparation and scanning protocol for computerised analysis of root length and diameter. Plant and Soil, v.218, p.185-196, 2000.

BREMNER, J.M.; MULVANEY, C.S. Nitrogen total. In: PAGE, A.L.; MILLER, R.H.; KEENEY, D.R. (Ed.). Methods of soil analysis. Madison: Soil Science Society of America, 1982. p.595-624. (ASA/SSSA. Agronomy, 9).

CHEVALIER, P.; SCHRADER, L.E. Genotypic differences in nitrate absorption and partitioning of $\mathrm{N}$ among plant parts in maize. Crop Science, v.17, p.897-901, 1977.

CHUN, L.; MI, G.H.; LI, J.; CHEN, F.; ZHANG, F. Genetic analysis of maize root characteristics in response to low nitrogen stress. Plant and Soil, v.276, p.369-382, 2005.

COQUE, M.; GALLAIS, A. Genomic regions involved in response to grain yield selection at high and low nitrogen fertilization in maize. Theoretical and Applied Genetics, v.112, p.1205-1220, 2006. 
FALCONER, D.S. Introduction to quantitative genetics. 2.ed. London: Longmans Green, 1981. 279p.

GALLAIS, A.; HIREL, B. An approach to the genetics of nitrogen use efficiency in maize. Journal of Experimental Botany, v.396, p.295-306, 2004.

GARNETT, T.; CONN, V.; KAISER, B.N. Root based approaches to improving nitrogen use efficiency in plants. Plant Cell and Environment, v.32, p.1272-1283, 2009.

LE GOUIS, J.; BÉGHIN, D.; HEUMEZ, E.; PLUCHARD, P. Genetic differences for nitrogen uptake and nitrogen utilization efficiencies in winter wheat. European Journal of Agronomy, v.12, p.163-173, 2000

LIMA, R.O. Herança e relação entre caracteres associados à morfologia de raiz e eficiência de uso de nitrogênio em milho. 2010. 35p. Dissertação (Mestrado) - Universidade Federal de Viçosa, Viçosa.

MACHADO, C.T. de T.; MACHADO, A.T.; FURLANI, A.M.C. Variação intrapopulacional em milho para características relacionadas com a eficiência de absorção e utilização de fósforo. Revista Brasileira de Milho e Sorgo, v.3, p.77-91, 2004.

MAIA, C.; DOVALE, J.C.; FRITSCHE-NETO, R.; CAVATTE, P.C.; MIRANDA, G.V. The difference between breeding for nutrient use efficiency and for nutrient stress tolerance. Crop Breeding and Applied Biotechnology, v.11, p.270-275, 2011.

MARANVILLE, J.W.; CLARK, R.B.; ROSS, W.M. Nitrogen efficiency in grain sorghum. Journal of Plant Nutrition, v.2, p.577-589, 1980.

MOLL, R.H.; KAMPRATH, E.J.; JACKSON, W.A. Analysis and interpretation of factors which contribute to efficiency of nitrogen utilization. Agronomy Journal, v.74, p.562-564, 1982.
OLIVEIRA L.R. Eficiência de uso de nitrogênio e atividade da nitrato redutase e glutamina sintetase em milho. 2009. 94p. Tese (Doutorado) - Universidade Federal de Viçosa, Viçosa.

PEREIRA, F.B. Relação entre caracteres determinantes na eficiência no uso de nitrogênio e fósforo em milho. 2011. 39p. Dissertação (Mestrado) - Universidade Federal de Viçosa, Viçosa.

RAUN, W.R.; SANDERS, D.H.; OLSON, R.A. Nitrogen fertilizer carriers and their placement for minimum till corn under sprinkler irrigation. Agronomy Journal, v.81, p.280-285, 1989.

RESENDE, M.D.V. de. Genética biométrica e estatística no melhoramento de plantas perenes. Brasília: Embrapa Informação Tecnológica, 2002. 975p.

RESENDE, M.D.V. de. Selegen-Reml/Blup: sistema estatístico e seleção genética computadorizada via modelos lineares mistos. Colombo: Embrapa Florestas, 2007. 359p.

SOUZA, L.V. de; MIRANDA, G.V.; GALVÃO, J.C.C.; ECKERT, F.R.; MANTOVANI, E.E.; DeLIMA, R.O.; GUIMARÃES, L.J.M. Genetic control of grain yield and nitrogen use efficiency in tropical maize. Pesquisa Agropecuária Brasileira, v.43, p.1517-1523, 2008.

TRACHSEL, S.; MESSMER, R.; STAMP, P.; HUND, A. Mapping of QTLs for lateral and axile root growth of tropical maize. Theoretical and Applied Genetics, v.119, p.1413-1424, 2009.

WALK, T.C.; JAMARILLO, R.; LYNCH, J.P. Architectural tradeoffs between adventitious and basal roots for phosphorus acquisition. Plant and Soil, v.279, p.347-366, 2006.

WORKU, M.; BÄNZIGER, M.; FRIESEN, D.; AUF'M ERLEY, G.S.; HORST, W.J.; VIVEK, B.S. Relative importance of general combining ability and specific combining ability among tropical maize (Zea mays L.) inbreds under contrasting nitrogen environments. Maydica, v.53, p.279-288, 2008.

Recebido em 14 de novembro de 2011 e aprovado em 24 de fevereiro de 2012

Pesq. agropec. bras., Brasília, v.47, n.3, p.385-392, mar. 2012 